\title{
Conduct responsible research to escape from predatory journals
}

\section{Mohammad Tariqur Rahman}

\section{KEYWORDS}

Predatory publication, publish or perish, number game, open access, article processing fees

\section{ABSTRACT}

Academics and researchers, willingly or unwillingly, continue to fall a "victim" to predatory journals. The entire cascade of moving forward in academia depends on one's ability to publish papers - as many as possible and in the shortest possible time. Such a requirement for a "number" persuades an academician or a researcher to race for an increased number of papers rather than to ensure the quality of the papers they want to publish. Thanks, but no thanks to the predatory journals - for providing a comfortable avenue for those papers to get published. An effective way out could be to train the academics and create awareness among them to conduct research following the codes of responsible research. Policymakers may also need to consider adopting policies that will not force their academic and research staff to race against time and compromise the codes of responsible research.

To the surprise of many of us, the recent Beall's list of the potential predatory publishers named more than 200 journals that are indexed in Scopus - a prestigious database maintained by Elsevier. Scopus indexing journals are given due credits for securing jobs or promotion by many academic and research institutions. There are other lists of predatory journals too [1].

Predatory journal (or their publishers) is defined as "entities that prioritize self-interest at the expense of scholarship and are characterized by false or misleading information, deviation from best editorial and publication practices, a lack of transparency, and/or the use of aggressive and indiscriminate solicitation practices" as defined earlier [2].

Needless to say, lecturers and researchers are the primary prey of predatory journals. Unlike the predatory relationship in the jungle, predatory journals seem to have mutual, commensal, or even symbiotic relationship with their preys in academia.

Academics and researchers, willingly or unwillingly, continue to fall a "victim" of predatory journals. Among the reasons given by 300 authors who published their papers in 50 listed predatory

Faculty of Dentistry, Universiti Malaya, Kuala Lumpur 50603, Malaysia. email: tarique@um.edu.my journals are: (i) predatory journals provide the services that are desired, (ii) predatory journals are an alternative route to avoid rejection by the Western journals, (iii) the authors have insufficient training in research methodology and reporting therefore, are unable to submit their manuscript to high-profile journals, and (iv) list of 'predatory' journals are not universal [3]. "Publish or perish" policy was also spelled out as one of the factors for academics and researchers to choose predatory journals [3].

Countries with a medium level of economic development with emphasis on R\&D, especially in Asia and North Africa, tend to be most susceptible to predatory publishing. Researchers from oil-rich Middle Eastern countries also appear to become easy prey [4].

In academia, recognition grows with the number of papers published by an individual. Albeit publications in high profile journals is the major deciding factor to fathom that recognition. Yet, a big number of publications is needed for jobs, promotions, and most of all to secure research grants. Before facing the paradox of "having track records of research to secure a research grant", one would struggle to get a job, thereafter, winning the race for promotion. In that race, focusing on the quality is often compromised. 
No doubt, running meaningful research is the right way to get more publications. However, it is easier said than done. The entire cascade of moving forward in academia depends on one's ability to publish papers - as many as possible and in the shortest possible time. Again, there is a requirement of having a certain number of publications before a student becomes eligible for a postgraduate degree (Masters or PhD). All together it is a race running against time (deadline).

Such a requirement for a "number" persuades many to race for an increased number of papers rather than to ensure the quality of the papers they want to publish. Thanks, but no thanks to the predatory journals - for providing a comfortable avenue to those papers to get published. Or else, getting a job or a promotion could have been more challenging especially in those institutions that are counting numbers only.

However, such mutual existence of predatory journals and their prey represents merely the boundary of the wilderness. Editorial team members of most predatory journals are none other than academicians - that also adds to their credit during job hunting or promotion. Therefore, it is unexpected that the academicians had to hath the scorn as they are the ones who doth the scathe.

Furthermore, it is kind of open secret that to publish a paper in a journal with a reasonable impact factor which offers open access or asks for article processing fees. That reflects a "degree of predation" in scientific communication.

Some aggressively predatory journals prey on victims without any quality while others hunt for victims with a certain level of quality. In other words, journals target the "vulnerable" prey depending on their own quality and the quality of the work they publish.
Perhaps this is one of the reasons for not being able to set a boundary to outcast the actual predatory journals.

With the increasing number of predatory journals having varying degrees of predation, it is unlikely to bring an end, at least in the near future, to this "symbiotic" relationship between the predators and prey in academia.

An effective way out could be to train the academics and create awareness among them to conduct research following the codes of responsible research [5] that includes but is not limited to: (i) scientific integrity to address basic technical competence (including experimental design), data manipulation, falsification, fabrication, and unintentional bias; (ii) collegiality to address authorship, data sharing, timely publishing, plagiarism, peer review, and confidentiality; (iii) protection of human subjects; (iv) animal welfare; (v) institutional integrity to address conflict of interest and commitment, regulatory compliance, data retention; and (vi) social responsibility to address research priorities, fiscal responsibility, public service, environmental impact, and domains of forbidden knowledge.

Policymakers may also need to consider adopting policies that will not force their academic and research staff to race against time and compromise the codes of responsible research.

\section{ACKNOWLEDGEMENT}

I wish to acknowledge the encouragement and support from Muhammad Manjurul Karim (University of Dhaka) and Rathna Devi Vaithilingam (University of Malaya).

\section{DECLARATION OF INTEREST}

The author reports no conflict of interest. The author alone is responsible for the content of this article.

\section{REFERENCES}

1. Cukier S, Helal L, Rice DB, Pupkaite J, Ahmadzai N, Wilson M, et al. Checklists to detect potential predatory biomedical journals: a systematic review. BMC Med. 2020; 18(1):104. [Dol: 10.1186/s12916-020-01566-1]

2. Grudniewicz A, Moher D, Cobey KD, et al. Predatory journals: no definition, no defence. Nature. 2019; 576:210-2.

3. Kurt S. Why do authors publish in predatory journals? Learn Publ. 2018; 31(2):141-7. [Dol: 10.1002/leap.1150

4. Macháček V, Srholec M. Predatory publishing in Scopus: evidence on cross-country differences. Scientometrics. 2021; 126(3):1897-921. [Dol: 10.1007/s11192-020-03852-4]

5. Pimple KD. Six domains of research ethics. A heuristic framework for the responsible conduct of research. Sci Eng Ethics. 2002; 8(2):191-205. 